\title{
Conditional transmissions, a strategy for highly dynamic vehicular ad hoc networks
}

\author{
Bertrand Ducourthial, Yacine Khaled and Mohamed Shawky* \\ Heudiasyc lab. UMR CNRS 6599, Université de Technologie de Compiègne \\ B.P. 20529, F-60205 Compiègne CEDEX, FRANCE \\ Email: firstname.name@hds.utc.fr
}

Technical report, Heudiasyc Lab., June, 10th 2006

\begin{abstract}
Many solutions have been developed for routing messages in ad hoc networks. However, few of them are efficient when the network is highly dynamic. Indeed, when the dynamic is high, building a routing table, discovering and maintaining a route or localizing a node is a great challenge. This topic is currently attracting attention with vehicular ad hoc networks (VANET), a special case of highly dynamic networks. VANET may allow to enhance road safety, and to develop new driver or passengers oriented services.

In this paper we present a novel approach for routing in highly dynamic networks, relying on conditions-based communication. Instead of transporting addresses (or positions), a message is sent with some conditions used for retransmission or reception. Thanks to the dynamic evaluation of the conditions, we show that this solution can efficiently support the high dynamic of vehicular networks. We also analyze the performances of OLSR, Fast OLSR, AODV, and GAMER on several traffic road scenarios.
\end{abstract}

\section{Introduction}

An ad hoc network is generally composed of nodes connected by wireless links. Usually, the topology of such a network is not planed. This is particularly the case when the nodes are mobile. In order to route the messages in such a network, any node can retransmit the messages to their destinations.

Many works have been done to design ad hoc routing algorithms, especially to deal with the node's mobility: updating some routing tables (pro-active algorithms), discovering some routes (reactive algorithms), using geographical

\footnotetext{
*Part of this work is funded by the regional grant "Pré-visibilité de routes" of the Region de Picardie (France).
} 
informations (geocast algorithms), detecting some stable structures (clusters), using the node's movements to transport the messages...

Besides the node's mobility, the algorithms have to take into account the node's dynamic: a node may appear or disappear in a neighborhood due to some power failures, obstacles, etc. An ad hoc network is highly dynamic if its topology is continuously and very frequently changing (due to node's mobility and node's dynamic) in such a way that nodes localization is never stable, either locally or globally. Routing messages in such a network is a great challenge.

This subject attracts many interest in particular for developing inter-vehicles networks. Among the applications, we can quote automatic driving, road safety enhancing by propagation of emergency alerts, and novel driver and passengers services. Every year in the European Union, about 40.000 people are killed on the roads, with around 1.7 million people incurring several injuries. Similar statistics have been estimated in the United States [12]. Generally, these accidents have been caused by faulty driver behaviors, bad weather conditions or mechanical problems. Hence, the inter-vehicles communications may help to anticipate the road accident and improve the road visibility [5]. Besides the safety applications, and when the bandwidth is not required by such applications, some entertainment applications may be deployed [14].

A Vehicular Ad hoc NETwork (VANET) is characterized by a strong mobility of the nodes, a high dynamic of the topology, a significant loss rate and a very short duration of communication. For example, with 250 meters communication range, two vehicles that cross each other at $110 \mathrm{~km} / \mathrm{h}$ can communicate only during 8 seconds. Our experiments on the road showed that this allows to send 800 KBytes with UDP over IEEE 802.11b. However the dynamic of such a network requires specific solutions for multi-hop communication in a convoy.

In this paper, we introduce the conditional transmissions. Instead of transporting addresses or positions, a message is sent with some conditions used for retransmission or reception. Thanks to the dynamic evaluation of the conditions, the conditional transmissions can efficiently support the high dynamic of the VANET. A stand-alone implementation has been developed to perform tests on the road with several vehicles. Moreover an implementation in ns-2 allowed to study the performances with many vehicles and different traffic scenarios. The performance study shows the interest of such an approach compared to classical routing algorithms.

In Section 2, a synthesis of the previous work related to routing algorithms in ad hoc networks is presented. The impact of the high dynamic is analyzed, as well as the intrinsic limitations of the main routing protocols families. A new approach of the communications in highly dynamic networks is also sketched. In Section 3, the conditional retransmissions technique is detailed, as well as some implementations. In Section 4, the performance study based on ns-2 simulations is presented. Finally, some concluding remarks end the paper. 


\section{Dealing with high dynamic}

\section{$2.1 \quad$ Related work}

In this section, the related work in routing algorithms is presented; a special focus is done on the high dynamic vehicular ad hoc networks.

\subsubsection{Topological routing protocols}

In a topological routing algorithm, the messages are routed with the help of the network topology, considered as a (moving) graph. The proactive protocols (e.g., OLSR [9]) build preventively some routing tables, even if there is no message to route. To the contrary, the reactive ones (e.g., AODV [7]) determine a route to a destination only on requests.

With OLSR, a node sends periodical some messages (Hello, Topology Control) to find its one-hop neighbors. It knows its two-hops neighbors through its neighbor's responses. Based on these informations, each node regularly determines its multipoint relays (MPR) as a subset of its one-hop neighbors allowing to reach its two-hops nodes. The messages are routed by using the MPR. With AODV, the route discovering is done by means of broadcast messages (route request RREQ). When it is reached, the destination replies by some unicast messages (route reply). The messages are then sent with unicast messages along the route. When it is broken, the route has to be rebuilt by means of special control messages.

Note that with OLSR the traffic overhead needed to maintain the routing tables increases with the dynamic, because the neighborhoods are always changing. This lead to an unstable situation when the network dynamic increases: while the table precision decreases, the construction overhead increases. Similarly, for reactive routing algorithms, the flooding needed to discover the routes may be less efficient when the network dynamic increases. Moreover the durability of the routes decreases. And the more the dynamic is large, the more the routes constructions need many control messages. Hence, while the route construction requires more messages, the route duration decreases.

Some works deal with the improvement of both OLSR and AODV in highly dynamic networks. In Fast OLSR [4], the frequency of the hello messages is adapted to the dynamic (by means of thresholds), and a small number of MPRs are selected to maintain the connectivity with other nodes in the network. In [13], the authors propose to add some geographical informations in the routing request packets of AODV. Such position related information can be used to avoid the topology limitations, particularly when it is moving.

\subsubsection{Geographical routing protocol}

The geographical routing protocol mainly rely on the informations given by some GPS receivers (one per node). Note that such an equipment is now cheap and common in vehicles. The geocast protocols (e.g., GAMER, LBM [23]) address all 
the mobiles belonging to an area. To the contrary, the position based protocols (e.g., LAR, DREAM, GPSR [24]) address a single node.

GAMER defines a sufficiently large area in order to take into account the node's mobility. This area can then be resized in case of success. LBM uses a fixed area but can also select the destination with the distance. With GPSR, a node selects its closest to the destination neighbor as the next hop.

Note that, when the dynamic increases, the positions are not stable, and the receiver position will no longer be valid when the message arrive. The position inaccuracy may lead to some performances issues. To deal with the mobility, the geographic area defining the destination can be increased, but this also increases the number of involved nodes and leads to bandwidth wasting.

\subsubsection{Hierarchical routing protocols}

With the hierarchical routing protocols, the network is composed of several clusters. A cluster is defined as a connected set of nodes sharing some characteristics, in order to remain stable as long as possible. The message propagation is done from clusters to clusters by means of gateway nodes. These nodes are in the transmission range of several cluster heads. Several levels of clusters may be defined (e.g., HSR [15]).

The hierarchical approach has been adapted to the topological routing protocols (e.g., CBRP [16], HSR [15]) or to the geographical routing protocol (e.g., ZHLS [17], GeoGRID [23]).

Note that the hierarchical protocols try optimize the resources usage. However the overhead needed to build such clusters increases with the network dynamic, while the clusters are less and less stable. In the VANET context, the algorithms may take into account traffic road characteristics in order to increase the life-time of the clusters [3, 25, 30, 21, 2]. Nevertheless to our opinion, a routing solution should not rely entirely on clusters, because in some situations, they do not exist. Instead, clusters should be used (when they exist) to enhance some routing strategies by limiting the number of re-transmitters for instance.

\subsubsection{Movement-based routing protocols}

In the movement-based routing protocols, the messages progress towards the destination by means of node's movements. Hence, a node may carry some messages until it meets their destinations $[19,2]$. The message propagation can rely on some epidemic or random schemes [29] or can rely on some subgraph of the entire network. Such a structure is similar to a backbone in fixed networks, but needs to evolve in dynamic networks. The evolutions of the structure can be controlled as in [20] or [8] with the so-called support-based routing. Some optimizations can be performed when the context is known [10]. For instance, in [31] the authors exploit the predictable vehicles mobility. However, to our opinion a routing solution should not rely entirely on the movement of the nodes because it is not sufficient nor practicable in all the situations. In VANET 
networks, the vehicles cannot be deviated in the aim of ensuring the routing. Instead such techniques may be used to improve the performances of more general routing strategies.

\subsection{A new approach for highly dynamic networks}

\subsubsection{Avoiding the addresses}

Our team is involved in the development of an embedded platform allowing to perform some tests on the roads [6]. Besides the software communication core, we develop distributed applications such as alert diffusion, road foreseen (visibility, obstacles...), distributed entertainments (talks, games...). This bottom-up approach shows that very few VANET applications need to establish a unicast communication.

Indeed, the receiver is often designated by some kind of conditions, such as "those who are behind the sender" (they will for instance encounter the hot spot"), "those who are in front of the sender" (they could for instance send some informations related to the road), "those who are a bridge towards another network" (such as the infrastructure access points, that could relay the message to wired network), "those who are in a given geographic area" (around a hot spot such as smog), "those who can offer a specific service" (service discovery), and marginally (but still a condition) "those who have the identity x" (a single receiver designated by its identity)

In fact, in a highly dynamic network, nodes can be designated by means of identities or conditions, but to our opinion it seems hazardous to designate them by means of network addresses. An identity is unique and can be randomly chosen to preserve privacy of the drivers. It is used only to distinguish two cars. To the contrary, a unicast address gives two kind of information: uniqueness identity of the node, and some informations related to the node's position in the network. However, managing the node's positions in the network seems too costly in a highly dynamic network. Indeed, both local (related to a neighborhood) and global (related to the entire network or landmark), either logical (eg. hierarchical address) or geographical (eg. GPS coordinates) positioning are difficult to maintain when the network dynamic increases. For instance, when sending a message towards a given position, chance are large that the receiver has moved, and that the information related to its position is out-of-date.

In order to deal with the highly dynamic network, we consider conditional addressing instead of network addressing, path maintaining instead of traditional unicast, and conditional transmissions instead of broadcast.

\subsubsection{Path maintaining}

The path maintaining problem consists in maintaining a communication that began when the receiver was in the neighborhood of the sender. A communication between two cars is generally initiated in such a situation, and not when the two cars are far from each other. Indeed, the identity - and even the presence 
in the network - of a distant vehicle is generally not known from the potential sender. And it would be too costly to make available such an information.

After some times, and because of the unforeseen turn of events in the road, the communication initiated in the neighborhood could need several hops to be achieved. This means that the path was initially of length 1 , and has to be maintained to allow the communication until a reasonable length. Note that the receiver is known by the sender because it was in its neighborhood (and only an identity is necessary). The communication can be maintained from neighborhood to neighborhood simply by using the identities.

\subsubsection{Conditional transmission}

Broadcast are common in VANET applications. However they should not flood the entire network in order to save the bandwidth. The conditional transmissions select the relay nodes by means of conditions, that allow to reduce the number of nodes involved. Moreover the receivers are also selected by means of conditions: only the nodes that fulfill the conditions will give the message to their application layers.

This scheme encompasses the geocast algorithms when the condition defines a geographic area ("being in a given area"). Note that it also encompasses the unicast communication when the condition defines a unique receiver ("having the identity $x ")$. However its main interest relies on the dynamic evaluation of the conditions performed at the message reception. By this way, this routing strategy better fits with the dynamic of the network. For instance, instead of designating the receivers as the cars which are in area $x$, the conditional transmissions designates those which are behind the sender and not farthest than $y \mathrm{~km}$ from it. The durability of such a condition-based addressing is larger than the classical addressing approach, even when it relies on geographical position (such as area $x$ ).

This solution can be seen as an intermediate solution between static addressing in the header of the messages, and mobile agents that may lead to dynamic behaviors. The former solution is not well adapted to dynamic network. The latter may be not accepted due to some security issues. Here, the condition evaluation allows to deal with the dynamic in an efficient way, without compromise any security requirement.

Note that the conditional transmissions could be used for optimizing other routing strategies. However we present in this paper some results for a standalone implementation, described in the next section.

\section{Conditional transmissions}

In this section, we describe the conditional transmissions as well as our standalone implementation (called HOP) both in simulator and embedded distributed framework. 


\subsection{Overview}

The main idea of the conditional transmissions is to send two conditions with each messages, namely the upward condition (CUP) and the forward condition $(\mathrm{CFW})$. When a message is received by a node, it is given to the application layer if CUP is true and it is forwarded to the neighbors (wireless emission) when CFW is true.

The conditions concern some geographic informations (distance from the sender, geographic position or area...) or some time-related informations (delay since the emission, date, message duration...). They can also concern some trajectories-related informations allowing to determine whether a mobile is on the same trajectory than the sender or not. Of course, the conditions may also be related to the identity of some nodes (sender, receiver, relay) or the kind of messages for instance, while this seems less useful in practice. Moreover any combination of conditions can be used.

The trajectories related conditions are often useful in VANET applications. For instance they allow to address a message only to the vehicles that will encounter an accident, and not to those which are on the same road but in the opposite direction. In order to have an accurate evaluation of such conditions, we developed a specific trajectory matching algorithm, well adapted to the road linear characteristics. Actually, using just the GPS positions of the vehicles to tag the messages is not efficient as the vehicles would never have exactly the same road trajectory. Furthermore, the absolute error in the position would not be necessarily identical to all vehicles in close vicinity, even if they are covered by the same satellites constellation. So our matching algorithm logs the last ten GPS positions of a vehicle in a circular buffer. These data are added to the exchanged messages. At the reception, some perpendicular projections are computed with received GPS positions and the receiver's own last positions. Finally, the correlation between the received trajectory and the receivers own trajectory is evaluated by comparison of a weighted ratio of the heights to a predefined threshold. See also [28] for a similar approach.

\subsection{Performances discussion}

Besides the CUP and CFW conditions, some data are added to the messages, in order to evaluate these conditions. For instance, when one of the conditions is related to the distance from the sender, the geographical position of the sender is sent with the message. Hence, the header of a message contains two conditions and some data necessary for their evaluation. This leads to variable length headers, which are often larger than classical routing algorithms. For instance, the condition used is Section 4 relies on distance and on trajectory matching. The header is about 200 bytes (20 char for CUP and 20 for CFW, 10 doubles for the latitudes and 10 for the longitudes). For comparison, the header of an OLSR message is about 12 bytes plus the list of the MPR used to join the destination. And the header of the geocast algorithms such as GAMER or LBM is about 80 bytes. Note however that the performance study presented in 
the next section takes into account this overhead, and shows nevertheless very interesting results.

The processing time depends on the complexity of the conditions. However a previous study shows the impact of the inter-packets gap (IPG) in inter-vehicles wireless communications: a too short IPG gives a poor throughput because it leads to many collisions [18]. Hence, the processing time necessary to evaluate the conditions has generally no impact on the performances.

Designing the relay nodes by means of conditions implies that many mobiles in the same neighborhood may fulfill the CFW conditions. In this case, they could all resend the message, leading to some bandwidth wasting. To solve this problem, the conditions can take into account some local parameters and some random techniques. A condition such as "rand ()$<1 / \mid$ neighbor-hood|" could be added to the CFW condition for instance. But other already known solutions can also be used: the conditional transmissions are indeed complementary to other broadcast optimizations. For instance, if some clusters (see $\S 2.1)$ are available, the conditions may forbid any retransmission in the vicinity of a cluster head. Or each relay may wait a (random) delay before resending the message, except if a neighbor already re-sent it (lazy retransmission) [27]. To not distort the performance study presented in Section 4, we do not consider any such optimization in this paper.

\subsection{Implementations}

An implementation of a conditional transmissions service (called HOP) has been developed as part of our embedded distributed framework [6]. This framework is used for the purpose of tests on the road with some vehicles. We also implemented HOP under Network Simulator [26] for larger performance studies.

\subsubsection{Embedded implementation}

The core program of our platform (called airplug [1]) manages all inter-applications communications, either local to a vehicle or wireless between vehicles. It runs on Linux and is written in $\mathrm{C}$ for portability and efficiency purpose. On each vehicle, all the applications are launched by airplug, and communicate through it, simply by writing to their standard output and by reading on their standard input. These local applications can be written in any language, providing it allows to perform asynchronous reading of the standard input. A distributed application is composed of a set of such local applications, distributed over the vehicles, and communicating via airplug by means of a wireless protocol (IEEE 802.11 in our tests). HOP is implemented as such a distributed application, and is used by some other applications.

The conditional transmissions prototype HOP has been written in tcl/tk (for compatibility with ns-2, see hereafter). Consider a distributed application $A$ composed of local applications $A_{i}$ on each vehicles $i$.

1. If $A$ requires multi-hop communication, each $A_{i}$ needs to be registered 
by their HOP local instance denoted $\mathrm{HOP}_{i}$. This is done by means of a special message sent locally from $A_{i}$ to $\mathrm{HOP}_{i}$ via airplug.

2. To send a message $m$ to some applications $A_{j}$ on other vehicles, $A_{i}$ gives to $\mathrm{HOP}_{i}$ (via airplug) the message $m$, a forward condition $\mathrm{CFW}$ and an upward condition CUP. By this way, the application determines the kind of communications, such as selective broadcast behind the car (alert), 2-hops communication around the vehicle (passenger's entertainment...), etc.

3. Upon reception of such a request, $\mathrm{HOP}_{i}$ sends to the neighbor vehicles (via airplug, wireless communication) the message $m$, the CUP and CFW conditions and some additional data needed to evaluate the conditions if needed.

4. The wireless message is received by the local HOP instance on neighbor vehicles via airplug.

5. On a neighbor vehicle $j, \mathrm{HOP}_{j}$ evaluates the CUP condition (using the additional data if needed). If CUP is true, the message $m$ is sent to the local application $A_{j}$ via airplug (local communication).

6. Next the CFW condition is evaluated. If it is true, the message $m$, the CFW and CUP conditions and the additional data are sent to the neighbors via airplug. The additional data may be updated in case of relaydependent conditions.

Some sequence numbers prevent to process several times the same message. We currently develop some additional programs to forge context aware conditions that take into account traffic road characteristics and convoys when they exist. However the description of these applications is out of the scope of this paper. Non context aware conditions already give very interesting performance, as seen in the next section. Several experiments on roads have been done with success.

\subsubsection{On the road experiments}

The conditional transmission have been tested with success during experiments on the roads. Four vehicles were involved: one stopped and the others in a mobile convoy of three vehicles. All the $k$ milliseconds, an application on the first vehicle generates a sequence of $p$ messages. Depending on the inter-vehicles distance, the third vehicle receives the messages either directly from the first vehicle (one hop) or from the second vehicle (two hop). Depending on the upward conditions (e.g., distance related conditions), the second vehicle in the convoy exploits or not the messages while it retransmits them. When the conditions are related to the trajectories, the stopped vehicle does not consider the messages because it does not share the same trajectory as the convoy. 


\subsubsection{Network simulator implementation}

In order to study the performances of the conditional transmissions on a large number of vehicles, we also implemented them in ns-2 [26]. This has been done with a network layer structure: HOP is implemented as a routing agent (similarly to DSDV, AODV, DSR...), and can send packets toward the other layers. The sending and receiving code is written in $\mathrm{C}++$, while the condition checking is written in tcl scripts, allowing to share some codes between the embedded platform and ns-2. The ns-2 implementation allows to take into account several scenarios with many cars and to perform comparisons with other routing techniques. This is presented in the next section.

\section{Performance study}

The performances of the conditional transmissions has been studied by means of simulations with ns-2. Some comparisons have been done with five routing algorithms in 4 traffic road scenarios. We first describe the methodology before detailing the results.

\subsection{Simulation methodology}

The simulations have been done with ns version 2.28 [26]. The propagation model is the two-ray ground. This model simulates a direct communication until a given inter-vehicles distance, and a communication with a single reflection on the road if the distance is larger. Indeed, while it can be improved [11], the ns-2 two-ray ground propagation model gives results sufficiently close to our tests on the road for this comparison study. The communication range is uniform; for each vehicle, it is equal to $250 \mathrm{~m}$ while the interference range is equal to $500 \mathrm{~m}$.

We consider 20 vehicles per convoy. The inter-vehicles distance takes the successive values of 27, 50,61 and 72 meters, corresponding (in France) to the expected security distance ( 2 seconds) related to the legal limitation speeds 50 , 90,110 and $130 \mathrm{~km} / \mathrm{h}$ respectively.

The first vehicle of the convoy sends regularly some packets to the others, with a constant inter-packets gap (IPG). The IPG is a convenient way to specify the sending rate in a convoy [18]. It takes the successive values of 5.5, 11, 14, 20,30 and $50 \mathrm{~ms}$, corresponding to the sending rate of $2048,1116,804,562,375$ and $225 \mathrm{kbit} / \mathrm{s}$ respectively. The size of all the packets is equal to 1440 bytes.

All the wireless communications, either broadcast or unicast, are performed with a sending rate of $2 \mathrm{Mbit} / \mathrm{s}$. This prevents the grey zone phenomena [22]. Indeed, the IEEE 802.11 broadcast packets are always transmitted at a basic bit rate while the data packets can be sent at higher rates. Then the broadcasted packets (such as the RREQ messages in AODV) can reach more remote nodes than data packets. As a consequence, a node may create a routing table with some nodes that will be reached by data packets only if they are sent at the basic bit rate. 
The transport protocol is UDP, while the routing protocol is varying. The conditional transmissions technique (HOP) is compared with two proactive protocols (OLSR, Fast OLSR), one reactive protocol (AODV) and two geocasts (LBM, GAMER). For all the simulations, the forward condition CFW used by HOP is a simple combination of the trajectory matching (see Section 3.1) and the distance: a vehicle retransmits a message only if it is behind the leader vehicle of the convoy, and separated by at least $200 \mathrm{~m}$ of it.
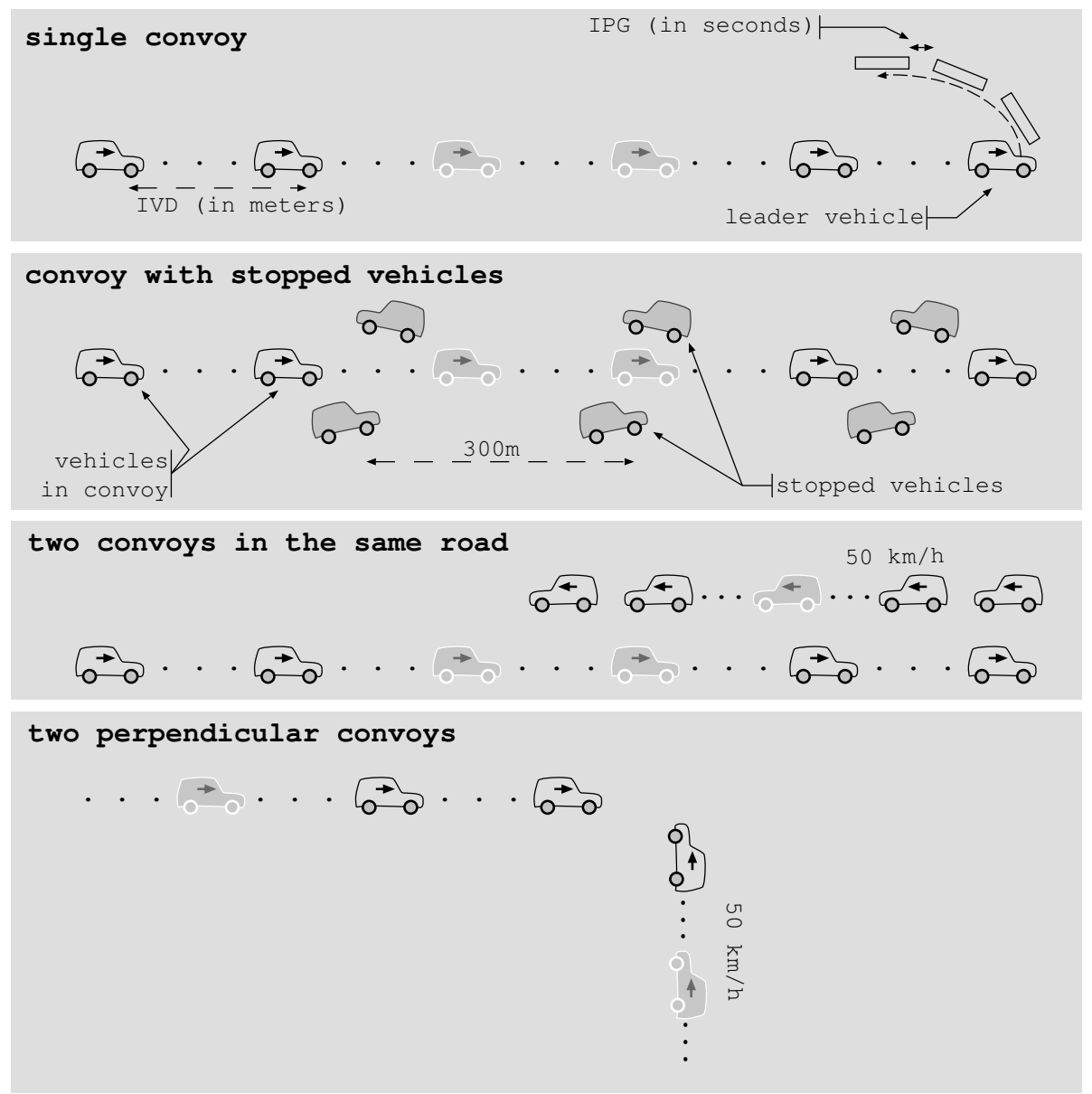

Figure 1: Different simulation scenarios.

Four traffic road scenarios have been considered (Fig. 1). The first one corresponds to a simple convoy with 20 vehicles. In the second scenario, some stopped vehicles are added each 300 meters on the both sides of the road. The third scenario consists in two convoys with opposite directions that crosses each other on the road. The relative speed of the second convoy varies. The communications are performed in the first convoy, but are affected by the vehicles 
of the second convoy. Finally, the last scenario consists in two convoys on two perpendicular roads, that crosses each other on a crossroad. The speed of the first convoy varies while the speed of the second is equal to $50 \mathrm{~km} / \mathrm{h}$ in order to maximize the perturbations duration with the first convoy. By simplicity, only rectilinear roads are considered (however the conditions used by HOP are robust to the shape of the roads).

Note that, in the first scenario, the vehicles are not mobile. Indeed, it is easily checked that the mobility has no influence (in this scenario) on the results of AODV, OLSR, Fast OLSR and HOP. Moreover, a fixed convoy allows to perform some comparisons with the geocast protocols GAMER and LBM. Indeed, these protocols define the receivers as those belonging to a fixed geographical area. In order to make some comparisons with the other protocols, the last vehicle of the convoy should receive the messages. Due to the convoy mobility, a large area should then be defined (in order that the last vehicle will be inside the area for the receptions). However, with such a large area, the messages will not reach the end of the convoy because many vehicles will enter in the area and will not retransmit them. Hence, the comparisons with the geocast protocols requires a fixed convoy. Such comparisons will only be done in the first scenario because the mobility factor is important for the other scenarios.

Each simulation corresponds to a transmission of 60 seconds, which is sufficient to stabilize the results. Twenty simulations have been done for each case and the displayed results are the averages. We did not observe any significant dispersion of the results (except for Fast OLSR in few cases). With 20 simulations per case, the evaluation of the four/six routing protocols on the four scenarios with different inter-packets gaps, relative speed and inter-vehicles distances leads to 2880 simulations.

Two kinds of performance criteria have been considered in order to represent different applications requirements. The first one is the end-to-end delay of the first packet (sent by the first vehicle of the convoy and received by the last vehicle). This criteria is important for applications such as alert diffusion. The second one is the ratio of the amount of data received by the last vehicle of a convoy to the amount of data sent by the first vehicle, expressed in percentage. The computations are based on the payload, meaning that the header sizes of each protocol are taken into account. This criteria gives some information related to the global throughput as well as to the end-to-end loss rate; it is important for inter-vehicles applications.

\subsection{Single convoy}

The objective of this first scenario is to compare HOP with five routing protocols (OLSR, Fast OLSR, AODV, LBM, GAMER) on a single convoy of vehicles.

The percentage of received data is shown in Figures 2 and 3 for small and large inter-vehicles distance (IVD) respectively. We observe very good performances of the conditional transmissions. 


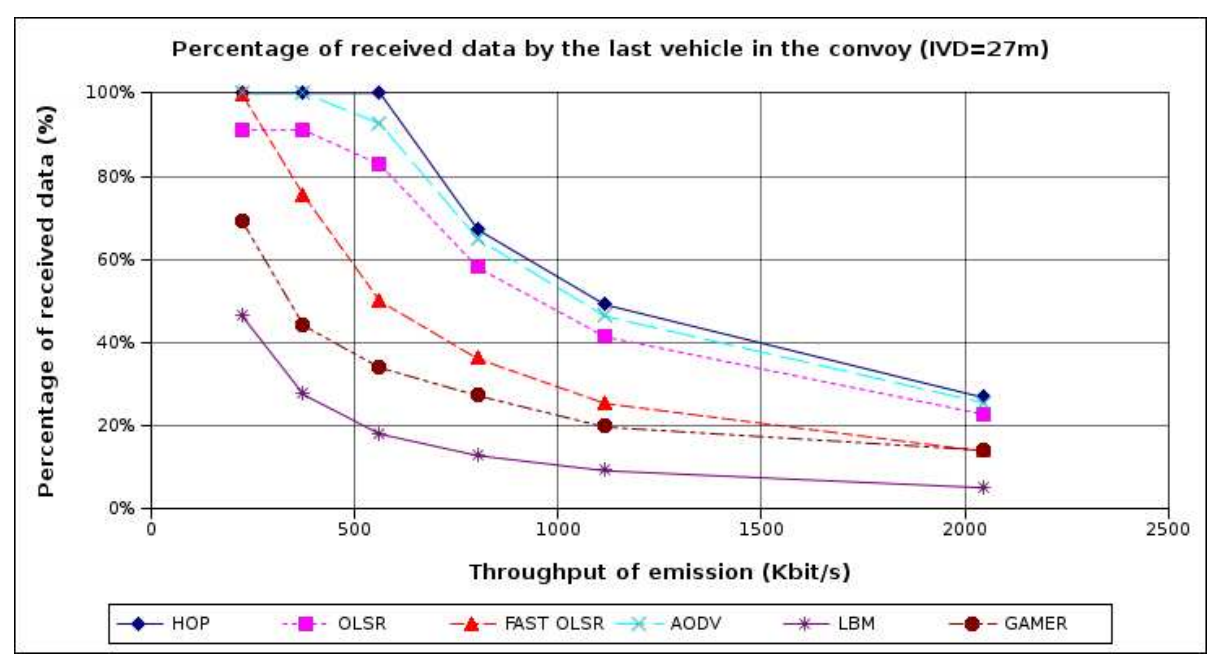

Figure 2: Percentage of received data in the single convoy with $\mathrm{IVD}=27 \mathrm{~m}$.

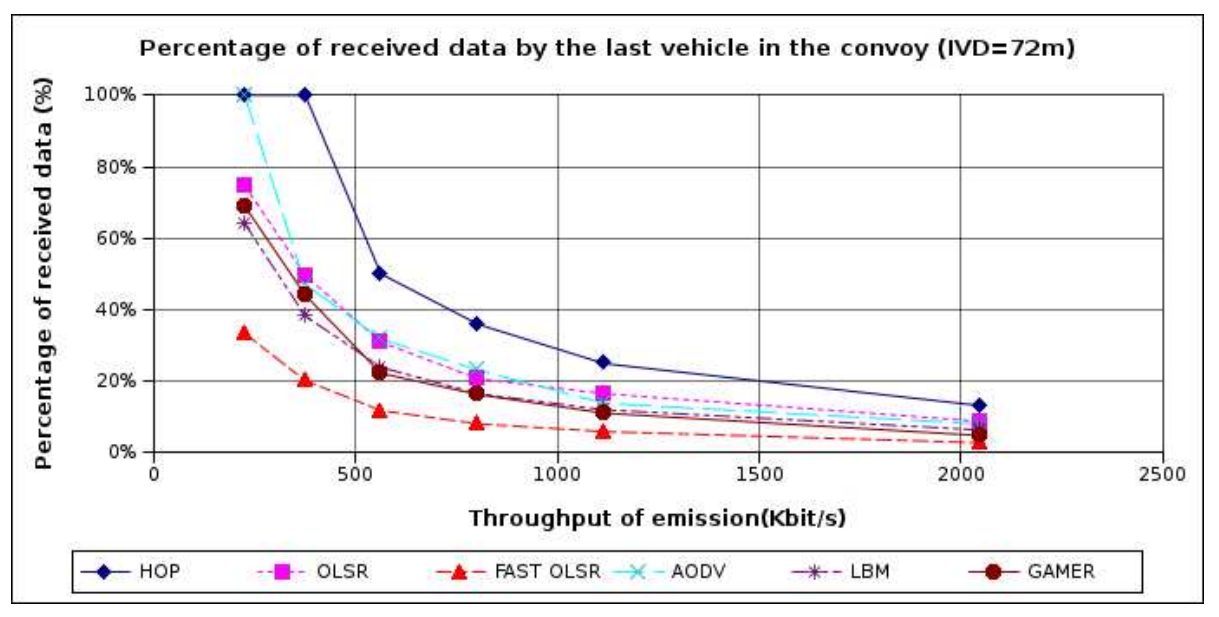

Figure 3: Percentage of received data in the single convoy with IVD=72 $\mathrm{m}$.

The performances decreases when the sending rate increases because when the sending rate increases, the inter-packets gap decreases and more collisions appear on the convoy (see [18]).

The percentage of received data for AODV is influenced by the inter-vehicles distance: performances are better with IVD $=27 \mathrm{~m}$ than with $\mathrm{IVD}=72 \mathrm{~m}$ (Figures 2 and 3). This is explained by the fact that a large inter-vehicles distances implies more hops to reach the end of the convoy.

It seems that OLSR and Fast OLSR are penalized when the vehicles density increases, that is when the inter-vehicles distance decreases. This can be 
explained by the fact that a high density increases the number of messages collisions, and then the delay for accessing the channel. The proactive protocols need to regularly send some control messages, and seem more affected by the density than others. The performance of OLSR and Fast OLSR increases with the convoy speed because the inter-vehicles distances also increases with the speed.

This is also the case of the geocast protocols such as LBM. Indeed, since all the nodes in the rectangle between the sender and the receiver retransmit the message, there is more collisions when the vehicle density is high. Hence, the performances decrease with the inter-vehicles distance IVD.

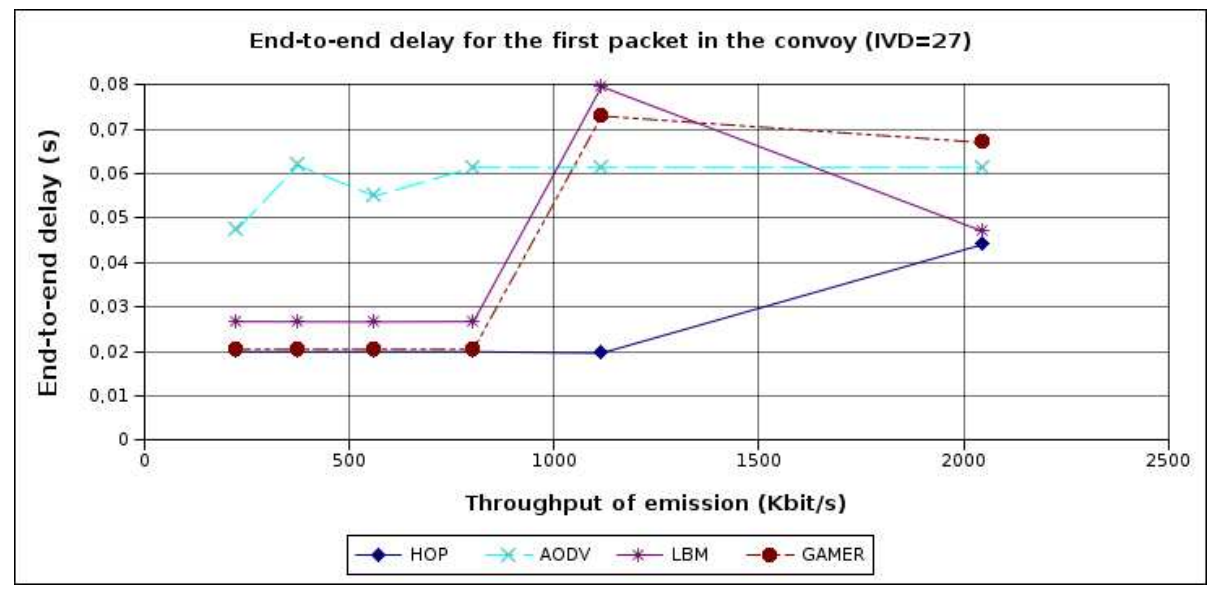

Figure 4: End-to-end delay for the first packet in the single convoy with $\mathrm{IVD}=27 \mathrm{~m}$.

The end-to-end delay of the first packet is shown in Figures 4 and 5. We also observe very good performances for the conditional transmissions. For instance, with an inter-packets gap of $0.014 \mathrm{~s}(800 \mathrm{kbit} / \mathrm{s})$, the delay is equal to $0.046 \mathrm{~s}$ with HOP and $0.103 \mathrm{~s}$ with AODV. The end-to-end delay is very important with OLSR (approximately $10 \mathrm{~s}$ with $\mathrm{IVD}=72 \mathrm{~m}$ ). This is due to the loss of TC messages, which are sent periodically by the nodes in order to define the MPRs. However, the delay for OLSR can be improved if the measures are done after the starting phase. For instance, after 20s the MPRs and routing tables become stable in this scenario, and we obtained a delay of $0.267 \mathrm{~s}$. But this remains large compared to AODV, and very large compared to HOP. The same phenomena has been observed with Fast OLSR. Hence, the end-to-end delay of the first packet is not displayed in the figure for OLSR and Fast OLSR in order to keep a precise scale. 


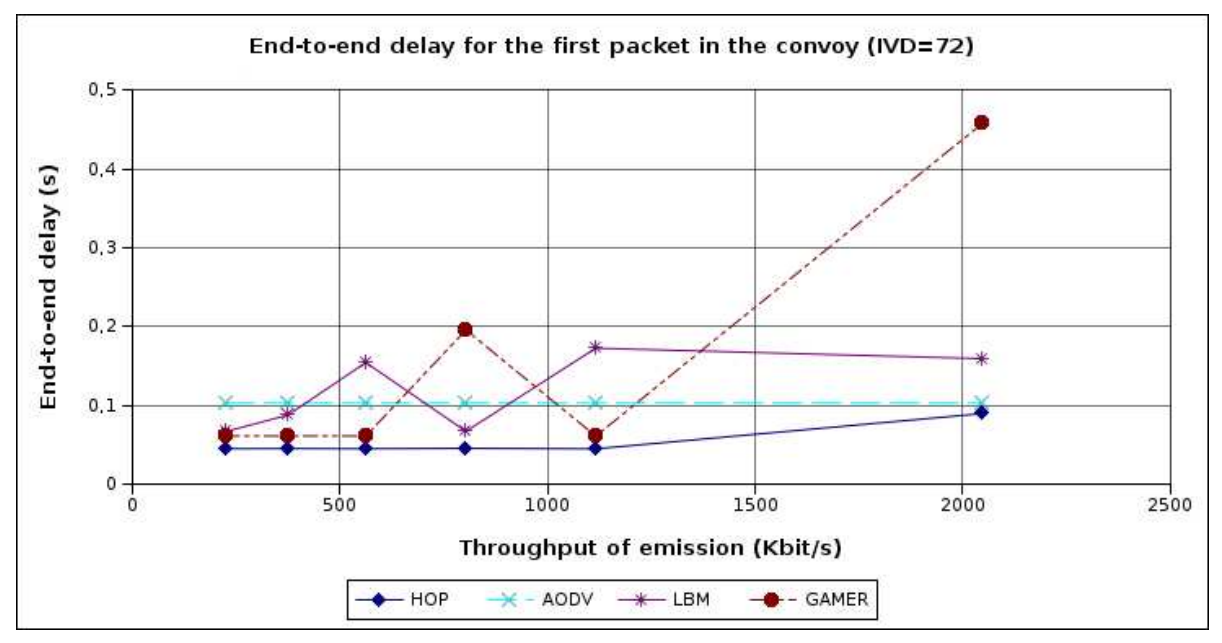

Figure 5: End-to-end delay for the first packet in the single convoy with $\mathrm{IVD}=72 \mathrm{~m}$.

\subsection{Convoy with stopped vehicles}

This scenario allows to study the impact of stopped vehicles along the road on the communications inside a mobile convoy.

We noticed the same phenomena related to the end-to-end delay for OLSR and Fast OLSR. Moreover, the end-to-end delay for AODV and HOP is not affected by the stopped vehicles. Hence, we only present the percentage of data received by the last vehicle in the convoy (Figures 6 and 7 ).

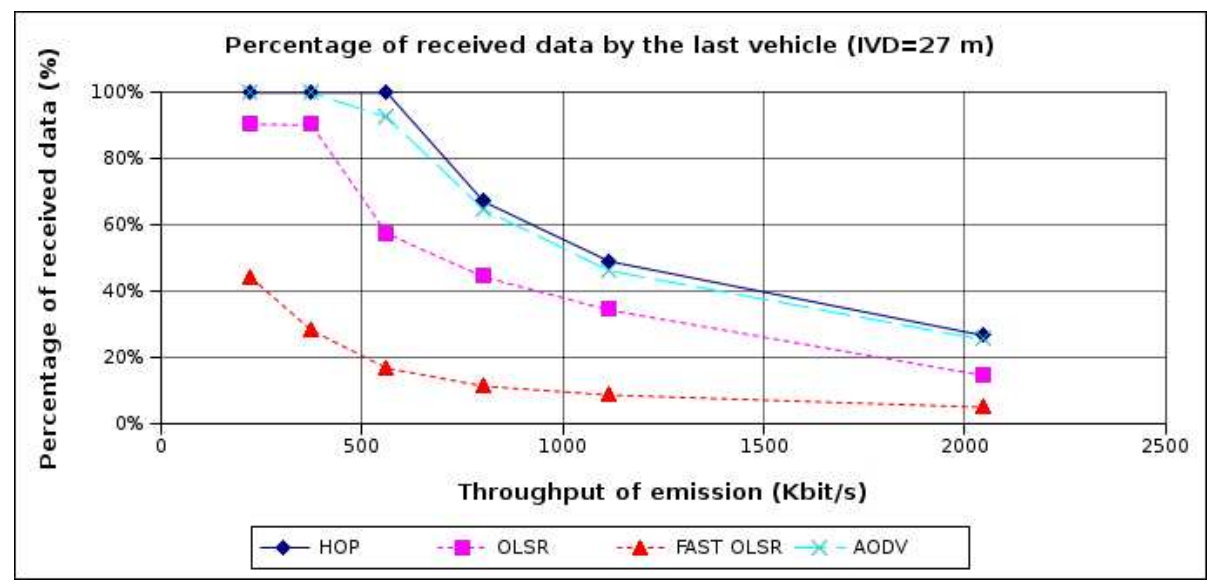

Figure 6: Percentage of received data in the convoy with stopped vehicles with $\mathrm{IVD}=27 \mathrm{~m}$. 


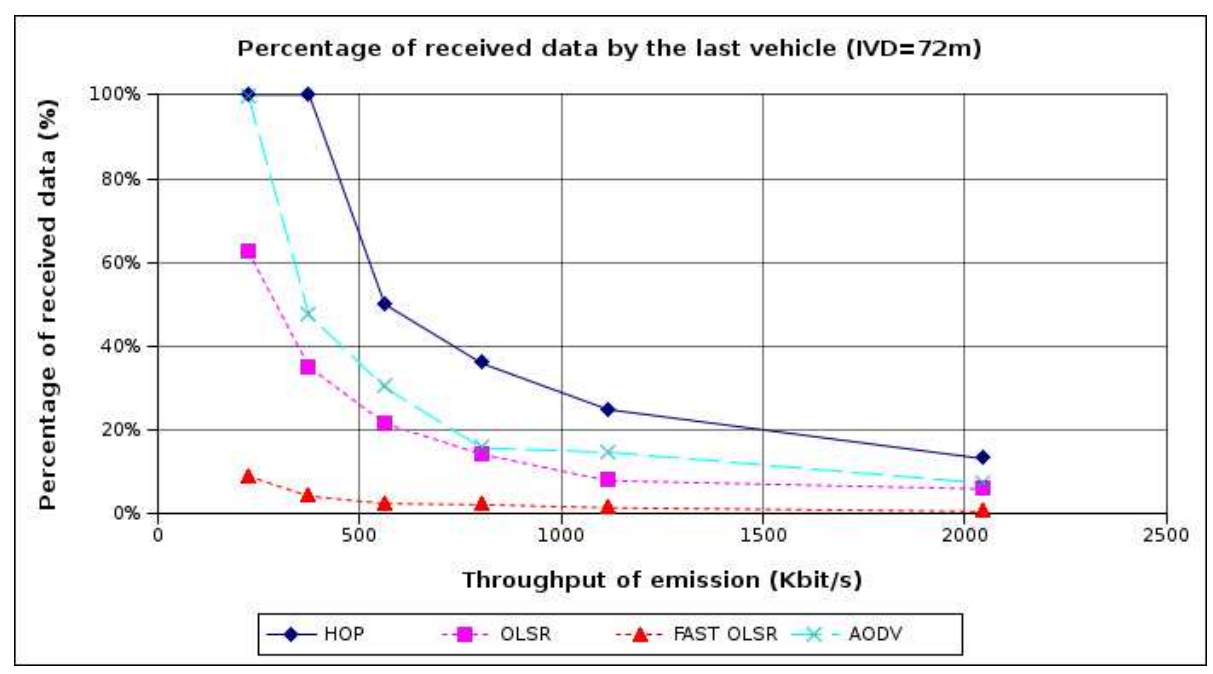

Figure 7: Percentage of received data in the convoy with stopped vehicles with $\mathrm{IVD}=72 \mathrm{~m}$.

The performances of HOP are not affected by the stopped vehicles because the forward condition $(\mathrm{CFW})$ is true only for the vehicles that belong to the convoy.

To the contrary, the more the inter-vehicles distance increases, the more AODV uses the stopped vehicles to route the messages. Indeed these vehicles become more often attractive to build a route when the inter-vehicles distance (IVD) is large. As a consequence, the percentage of routes breaking is larger when IVD increases. Note that this does not affect the delay of the first packet.

The following table gives the percentage of received data of the first and second scenarios, for an inter-vehicles distance of $72 \mathrm{~m}$, and an inter-packets gap of $0.014 \mathrm{~s}$. These results confirm that the performances of AODV, OLSR and Fast OLSR are affected by the stopped vehicles along the road, to the contrary of HOP.

\begin{tabular}{|c|c|c|c|c|}
\hline & OLSR & Fast OLSR & AODV & HOP \\
\hline Single convoy & $21 \%$ & $8 \%$ & $23 \%$ & $36 \%$ \\
\hline With stopped veh. & $14 \%$ & $2 \%$ & $15.7 \%$ & $36 \%$ \\
\hline
\end{tabular}

\subsection{Crossing convoys}

The objective of this scenario is to compare the performances of HOP, AODV, OLSR and Fast OLSR in a mobile convoy which crosses another one on the same road.

The same remarks related to the end-to-end delay still apply in this scenario. The Figures 8 and 9 illustrate the percentage of received data by the last vehicle in the convoy with 27 and $72 \mathrm{~m}$ of inter-vehicles distance respectively. 


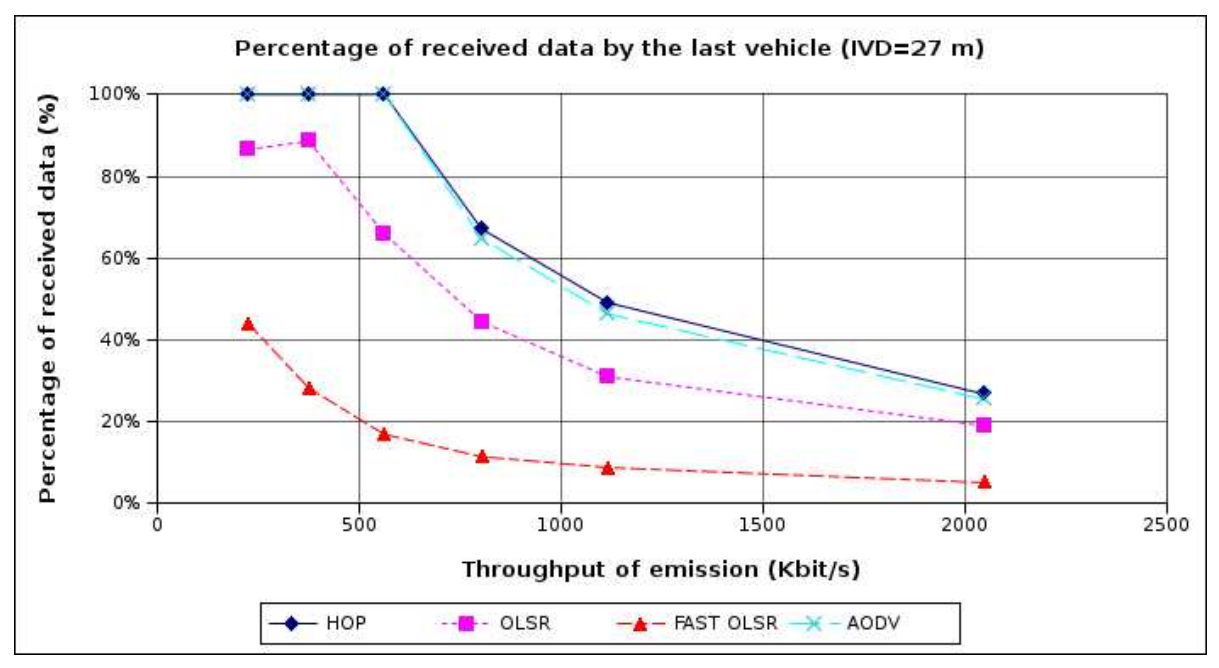

Figure 8: Percentage of received data in the convoy with a crossing convoy and with $\mathrm{IVD}=27 \mathrm{~m}$.

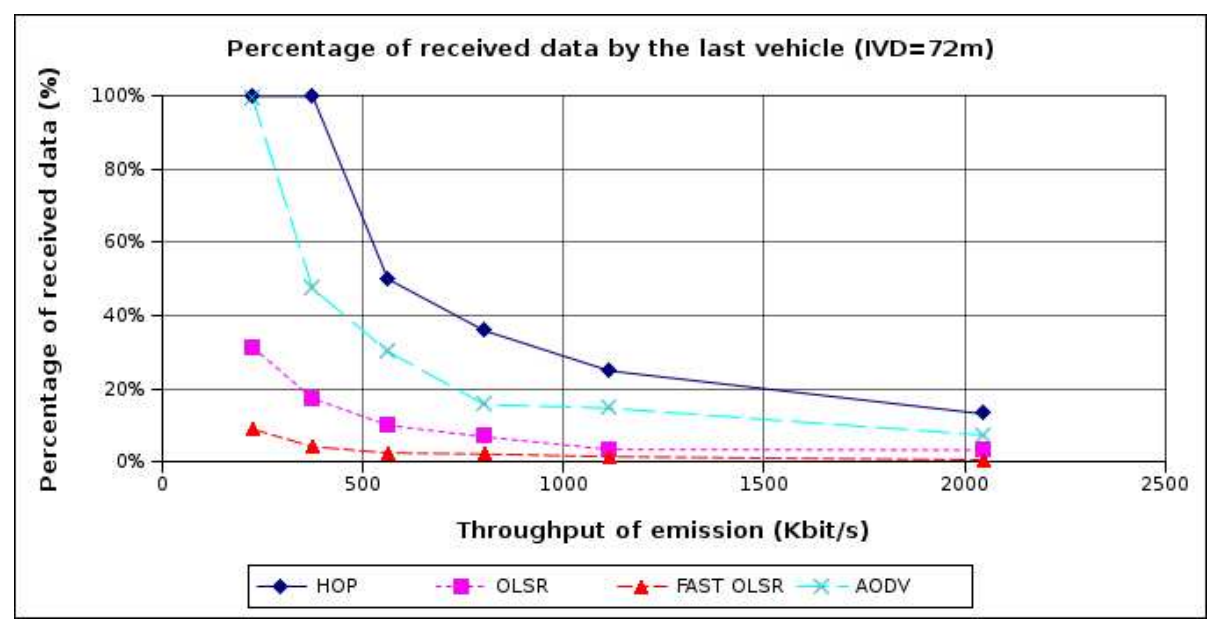

Figure 9: Percentage of received data in the convoy with a crossing convoy and $\mathrm{IVD}=72 \mathrm{~m}$.

The performance of OLSR are bad comparatively to other protocols. This can be explained by the density increased by the crossing convoy, and by the control messages collisions.

The performance of AODV is affected by the second convoy. Indeed some (unstable) routes are built with the vehicles of the second convoy, increasing the number of routes breaking.

The performances of HOP are not disturbed by the crossing vehicles, because 
only the vehicles of the first convoy are involved, thanks to the CFW condition.

The following table gives the percentage of received data of the first and third scenario, for an inter-vehicles distance of $72 \mathrm{~m}$, and an inter-packets gap of $0.014 \mathrm{~s}$. These results confirm that the performances of AODV, OLSR and Fast OLSR are affected by the vehicles in the crossing convoy. This is particularly true for OLSR (21\% compared to $7 \%$ ) and Fast OLSR (8\% compared to $2 \%$ ). These results shows that only HOP is not affected by the crossing convoy.

\begin{tabular}{|c|c|c|c|c|}
\hline & OLSR & Fast OLSR & AODV & HOP \\
\hline Single convoy & $21 \%$ & $8 \%$ & $23 \%$ & $36 \%$ \\
\hline With crossing convoy & $7 \%$ & $2 \%$ & $15.7 \%$ & $36 \%$ \\
\hline
\end{tabular}

\subsection{Perpendicular crossing convoys}

The objective of this scenario is to compare the different protocols on a principal convoy of vehicles with a perpendicular crossing convoy.

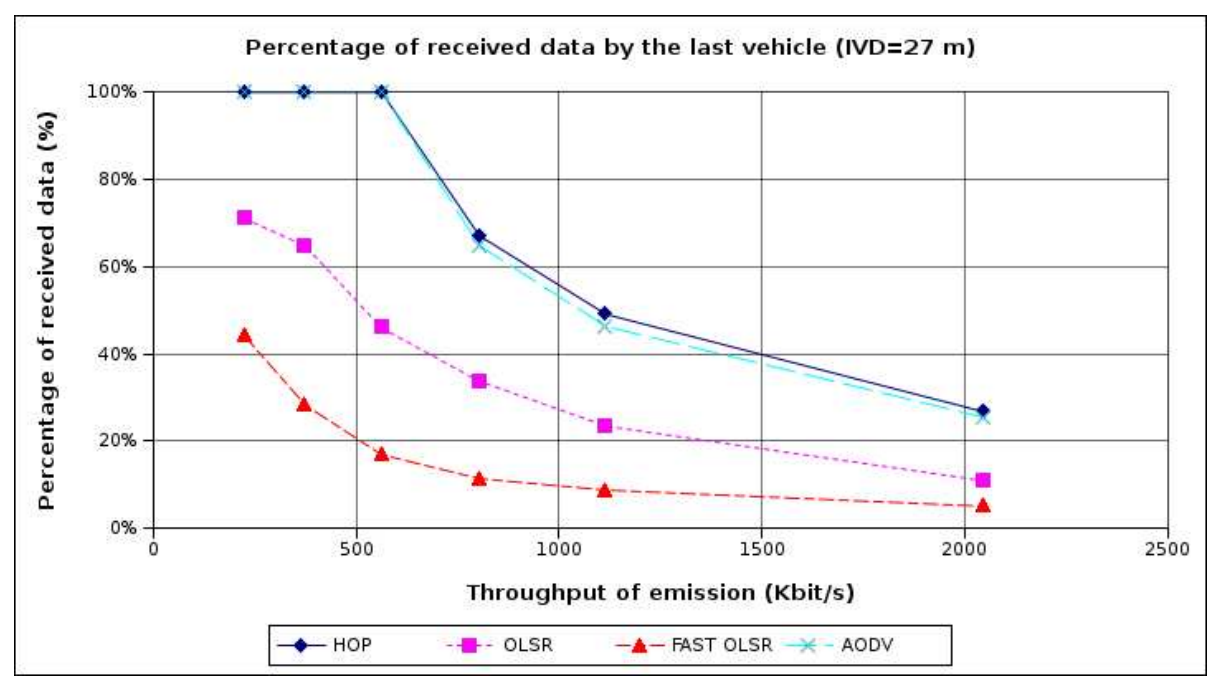

Figure 10: Percentage of received data in the convoy with a perpendicular crossing convoy and with $\mathrm{IVD}=27 \mathrm{~m}$.

The same remarks related to the end-to-end delay still apply in this scenario, and we only present the percentage of data received by the last vehicle in the convoy with an inter-vehicles distance of 27 and $72 \mathrm{~m}$ (Figures 10 and 11).

The performances of OLSR in this scenario are better than in the previous one. This may be explained by the fact that the vehicles density is less important with a perpendicular convoy than with a parallel convoy. 


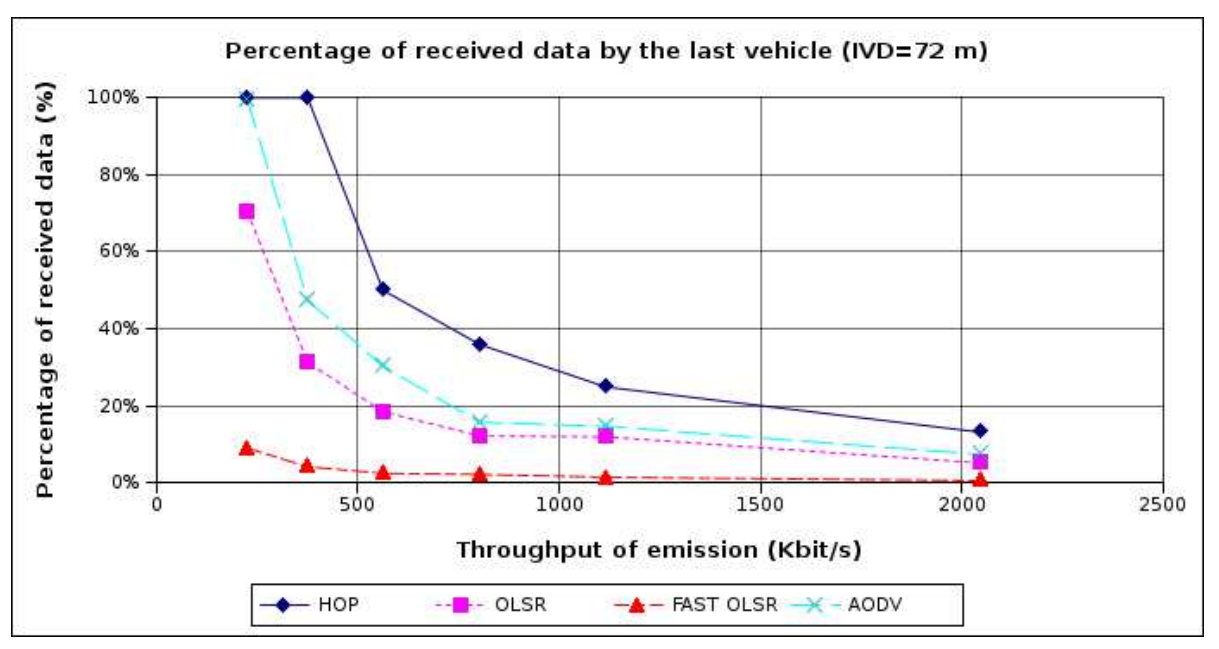

Figure 11: Percentage of received data in the convoy with a perpendicular crossing convoy and with $\mathrm{IVD}=72 \mathrm{~m}$.

The following table gives the percentage of received data of the first and fourth scenario, for an inter-vehicles distance of $72 \mathrm{~m}$, and an inter-packets gap of $0.014 \mathrm{~s}$. These results confirm that the performances of AODV, OLSR and Fast OLSR are affected by the vehicles in the perpendicular convoy.

\begin{tabular}{|c|c|c|c|c|}
\hline & OLSR & Fast OLSR & AODV & HOP \\
\hline Single convoy & $21 \%$ & $8 \%$ & $23 \%$ & $36 \%$ \\
\hline With perp. convoy & $12 \%$ & $2 \%$ & $15.7 \%$ & $36 \%$ \\
\hline
\end{tabular}

\subsection{Conclusions}

In the four scenarios, HOP obtains better results than other protocols. The conditional transmissions allow to define some communications in a convoy without being affected by the road traffic. While the ad hoc network topology is highly dynamic, HOP always offers very acceptable performances for the applications, to the contrary of proactive, reactive and geocast routing algorithms.

By comparison, the other protocols suffer from the dynamic. Indeed, since the topology is very unstable, the routing tables are always inaccurate, and require many messages to be updated. Our simulations show poor performances for the proactive routing protocols (OLSR and Fast OLSR). On an other hand, reactive protocols (such as AODV) need to seek a route from the sender to the receiver. But the durability of the routes is low, and many control messages are needed to maintain the communication. The performances of the reactive protocols are better than the proactive ones, but can still largely be improved. And while the geocast routing protocols (such as LBM and GAMER) are adapted to send a message in a specific geographic area, they cannot efficiently be used to send a message to a highly mobile node. As a consequence, they are not really adapted to perform some communications in a mobile convoy. 


\section{Conclusion}

\subsection{Summary}

This paper deals with the communications in highly dynamic networks. Among such networks, the vehicular ad hoc networks (VANET) are currently attracting attention because they could allow to enhance road safety, and to develop new drivers or passengers oriented services.

Several works have been done to design routing algorithms in the ad hoc networks: topology-based either proactive or reactive, geographical, hierarchical, movement-based... However, building a routing table, discovering and maintaining a route, localizing a node, maintaining a cluster... remains a great challenge in a highly dynamic ad hoc network such as VANET. An ad hoc network is considered highly dynamic when its topology is very frequently changing in such a way that node's localization is almost never stable, either locally or globally.

In order to deal with such high dynamic networks, we propose in this paper a novel approach relying on conditional transmissions. Instead of transporting addresses or positions, a message is sent with some conditions used for retransmission or reception. Thanks to the dynamic evaluation of the conditions, our solution can efficiently support the high dynamic of the networks. A stand-alone implementation (called HOP) on our embedded distributed framework allows to perform tests on the road with several vehicles. Besides this prototype, an implementation in ns-2 enables to scrutinize the performances of HOP with many vehicles, with four different traffic scenarios and with several parameters values. Among the conditional transmission, one of the contributions of this paper is the performance analysis of some well known routing algorithms in these scenarios.

The simulation results show that the conditional transmissions offer better performances than (i) the proactive algorithms OLSR and Fast OLSR, (ii) the reactive algorithm AODV and (iii) the geocast algorithms LBM and GAMER. The end-to-end delay of the first packet is very short with HOP and is not affected by the traffic road scenario. The end-to-end ratio of received data to sending data is also not affected by the dynamic. It logically decreases when the inter-packets gap of the source decreases [18], but it remains very interesting.

\subsection{Future work}

Our study was concerned with a stand alone implementation of the conditional transmissions. We compared them with five well known protocols. Other comparisons could be done in the future with other protocols provided that they are available for network simulator. Moreover, the conditional transmission may be used in conjunction with other routing strategies. For instance, the conditions could be related to clusters when they exist. They also could complete the movement based protocols. And they could optimize some flooding in topology-based protocols (such as RREQ in AODV). This is one of the directions for future works.

A future work may also concern the study of this technique in other highly 
dynamic networks, not necessarily VANET. This may allow to determine some limits or some network requirements for a general purpose usage in highly dynamic networks.

Also, with a better knowledge of the traffic road, the conditions could be refined, and the communications optimized. For instance, when the convoy is not connected, it could be interesting to use some other external vehicles to ensure the communication in a sparse traffic. This means that better performances could still be obtained with some context aware conditions, forged with a program that takes into account the traffic road characteristics. This of course implies to determine these characteristics on-line. We are currently designing such programs, allowing to detect the presence of convoy and the traffic characteristics (city/highway traffic, chaotic/regular traffic, traffic jams and so one), and to forge some context-aware conditions for efficient communications on the roads.

\section{References}

[1] Airplug: http://www.hds.utc.fr/ ducourth/contrib/airplug.

[2] G. Allard, P. Jacquet, and B. Mans. Routing in extremely mobile networks. In Proceedings of 4th Annual Mediterranean Ad Hoc Networking Workshop, Île de Porquerolles, France, 2005.

[3] P. Basu, N. Khan, and T. Little. A mobility based metric for clustering in mobile ad hoc networks. In International Conference on Distributed Computing Systems Workshop, Mesa, Arizona, USA, 2001.

[4] M. Benzaid, P. Minet, and K. Agha. Integrating fast mobility in the OLSR routing protocol. In Proceedings of IEEE Conference in Mobile and Wireless Communications Networks, Stockholm, 2002.

[5] http://www.car-to-car.org.

[6] Caremba: http://www.hds.utc.fr/caremba.

[7] I. Chakeres and M. Belding-Royer. AODV routing protocol implementation design. In Proceedings of the International Workshop on Wireless Ad Hoc Networking (WWAN), Tokyo, Japan, 2004.

[8] I. Chatzigiannakis, E. Nikoletseas, and P. Spirakis. An efficient communication strategy for ad-hoc mobile networks. In Proceedings of the 15th International Conference on Distributed Computing (DISC), London, UK, 2001.

[9] T. Clausen, P. Jacquet, A. Laouiti, P. Muhlethaler, A. Qayyum, and L. Viennot. Optimized link state routing protocol. In Proceedings of IEEE International Multitopic Conference INMIC, Pakistan, 2001. 
[10] J. Davis, A. Fagg, and B. Levine. Wearable computers as packet transport mechanisms in highly-partitioned ad-hoc networks. In Proceedings of the 5th IEEE International Symposium on Wearable Computers (ISWC), Washington, DC, USA, 2001.

[11] N. Eude, B. Ducourthial, and M. Shawky. Enhancing ns-2 simulator for high mobility ad hoc networks in car-to-car communication context. In Proceedings of the 7th IFIP International Conference on Mobile and Wireless Communications Networks (MWCN 2005), Morocco, 2005.

[12] http://europa.eu.int/comm/transport/care/.

[13] T. Fukuhara, T. Warabino, T. Ohseki, K. Saito, K. Sugiyama, T. Nishida, and K. Eguchi. Broadcast methods for inter-vehicle communications system. In Proceedings of IEEE Wirelesss Communication and Networking Conference, New Orleans, 2005.

[14] M. Gerla. http://www.mobiquitous.org/2005/gerla.pdf.

[15] A. Iwata, C. Chiang, G. Pei, M. Gerla, and T. Chen. Scalable routing strategies for ad hoc wireless networks. IEEE Journal on Selected Areas in Communications, Special Issue on Ad-Hoc Networks, August 1999.

[16] M. Jiang, J. Li, and Y. Tay. Cluster based routing protocol (CBRP). Technical report, IETF, 2001. Internet draft.

[17] M. Joa-Ng and I.-T. Lu. A peer-to-peer zone-based two-level link state routing for mobile ad hoc net-works. IEEE on Selected Areas in Communications, vol. 17, no. 8:1415 1425, 1999.

[18] Y. Khaled, B. Ducourthial, and M. Shawky. IEEE 802.11 performances for inter-vehicle communication networks. In Proc. of th 61st IEEE Semianual Vehicular Technology conference VTC, Stockholm, 2005-Spring.

[19] T. Kosch. Technical concept and prerequisites of car-to-car communication. Technical report, BMW Group Research and Technology, 2002.

[20] Q. Li and D. Rus. Sending messages to mobile users in disconnected ad-hoc wireless networks. In Proceedings of the 6th annual international conference on Mobile computing and networking (MOBICOM), 2000.

[21] C. Lin and M. Gerla. Adaptive clustering for mobile wireless networks. IEEE Journal on Selected Areas in Communications, 1997.

[22] H. Lundgren, E. Nordstrom, and C. Tschudin. The gray zone problem in IEEE 802.11 b based ad hoc networks. ACM SIGMOBILE Mobile Computing and Communications Review, 6:104-105, July 2002.

[23] C. Maihfer. A survey of geocast routing protocols. IEEE Communications Surveys and Tutorials, 6, 2nd quarter 2004. 
[24] M. Mauve, J. Widmer, and H. Hartenstein. A survey on position-based routing in mobile ad hoc networks. IEEE Network Magazine, November/December 2001.

[25] B. McDonald and T. Znati. Design and performance of a distributed dynamic clustering algorithm for ad-hoc networks. In Annual Simulation Symposium, Seattle, WA, USA, 2001.

[26] Network simulator 2: http://www.isi.edu/nsnam/ns/.

[27] I. Stojmenovic, M. Seddigh, and J. Zunic. Dominating sets and neighbor elimination-based broadcasting algorithms in wireless networks. IEEE Transactions on Parallel and Distributed Systems, Vol. 13, No. 1:14-25, January 2002.

[28] M. Sun, W. Feng, T. Lai, K. Yamada, H. Okada, and K. Fujimura. GPSbased message broadcasting for inter-vehicle communication. In International Conference on Parallel Processing, Toronto, Canada, 2000.

[29] A. Vahdat and D. Becker. Epidemic routing for partially connected ad hoc networks. Technical Report CS-200006, Computer Science Dpt, Duke University, 2000.

[30] J. Yu and P. Chong. 3hbac (3-hop between adjacent clusterheads): a novel non-overlapping clustering algorithm for mobile ad hoc networks. In In IEEE Pacific Rim Conference on Communications, Computers and Signal Processing, Victoria, B.C., Canada, 2003.

[31] J. Zhao and G. Cao. VADD: Vehicle-assisted data delivery in vehicular ad hoc networks. In Proceedings of the 25th Conference on Computer Communications (INFOCOM), Barcelona, Spain, 2006. 